\title{
A rare development of tumoral calcinosis of the ear auricle
}

\author{
Sam Yong Lee, \\ Han Gyeol Lee, \\ Kwang Seog Kim, \\ Jae Ha Hwang \\ Department of Plastic and \\ Reconstructive Surgery, Chonnam \\ National University Medical School, \\ Gwangju, Korea
}

\begin{abstract}
Tumoral calcinosis is a condition characterized by deposition of calcium salts in the skin and subcutaneous tissue, commonly found around the joints. However, tumoral calcinosis of the auricle is extremely rare. We present the case of a 13-year-old boy with tumoral calcinosis of the helix of the ear auricle. A 13-year-old boy presented with a 10-year history of an enlarging mass on the left auricle. The mass was hard, non-tender, and non-compressible. The patient had no history of trauma. Complete surgical excision and pathological examination of the specimen was performed. The final diagnosis of the excised mass was tumoral calcinosis. After 9 months of follow-up, there were no signs of recurrence of the tumor and the patient was satisfied with the surgical results. Tumoral calcinosis of the auricle is extremely rare and may be misdiagnosed as other tumors. Pathological examination is essential for definitive diagnosis and complete surgical excision should be considered as the treatment of choice.
\end{abstract}

Keywords: Calcinosis / Ear neoplasms / Tumoral calcinosis

\section{INTRODUCTION}

Calcinosis cutis is a rare disease consisting of deposition of calcium salts in the skin and subcutaneous tissue and can be classified as dystrophic, metastatic, idiopathic, or iatrogenic [1,2]. Tumoral calcinosis is a special form of the idiopathic calcinosis cutis and was first named in 1943 by Inclan et al. [3,4]. Tumoral calcinosis presents as a painless, firm, tumor-like mass and is commonly found around the hip, shoulder, elbow, and knee joints [3]. However, tumoral calcinosis of the auricle is extremely rare. We present the case of a 13-year-old boy with tumoral calcinosis of the helix of the ear auricle.

\section{CASE REPORT}

A 13-year-old boy presented with a chief complaint of a grow-

Correspondence: Jae Ha Hwang

Department of Plastic and Reconstructive Surgery, Chonnam National University

Medical School, 42 Jebong-ro, Dong-gu, Gwangju 61469, Korea

E-mail: psjhhwang@daum.net

Received October 23, 2018 / Accepted November 20, 2018 ing mass on the helix of left auricle for 10 years (Fig. 1). It was hard and painless, with non-tender and non-compressible swelling. There was no history of trauma on the auricle or any family history of tumor disease, except for presence of keloid skin in the mother. Ultrasonography investigation revealed a $1.15 \times 0.7 \times 1.45-\mathrm{cm}$ heterogeneous hyperechoic mass on the helix of the left auricle (Fig. 2), which was clinically diagnosed as cartilage-origin benign neoplasm.

Under local anesthesia, complete surgical excision of the tumor was performed. A vertical incision was made through superior helix. The tumor was removed entirely by delicate dissection. The tumor appeared to be well circumscribed, hard, spherical mass measuring about $2 \mathrm{~cm}$ in diameter (Fig. 3).

The histopathological examination result showed that normal auricle structure was hardly visible, and extensive calcification was seen as a purple-blue, homogenous material. Dense hyalinized stroma surrounded the calcified material with formation of multiple cystic spaces and progressive calcification with eosinophilic debris (Fig. 4A). Foreign body giant cell reaction with calcification and epithelioid cell were also observed (Fig. 4B). 


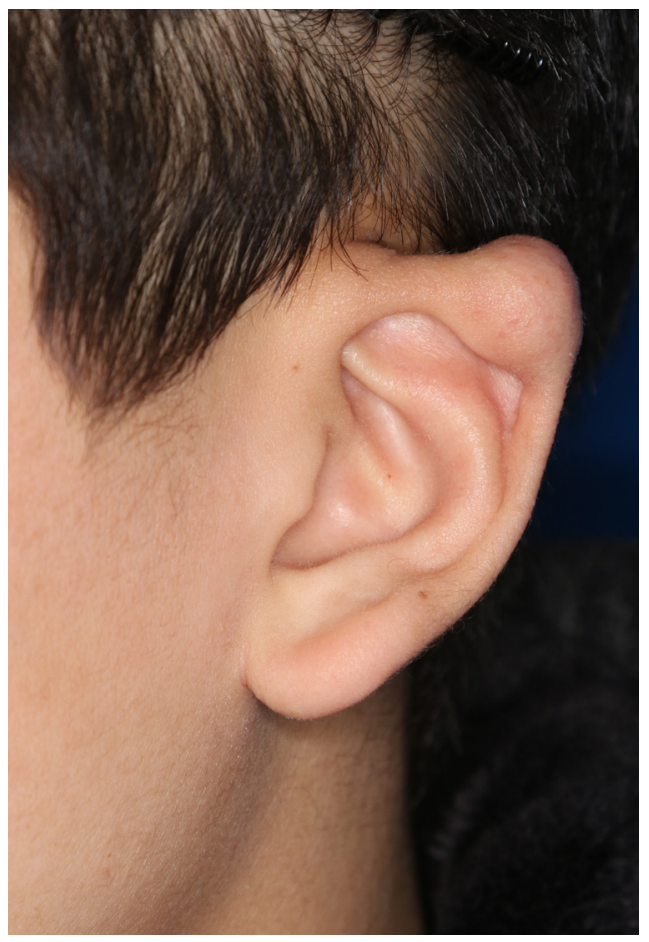

Fig. 1. Mass on the helix of the left auricle measuring approximately $2 \mathrm{~cm}$ in diameter.

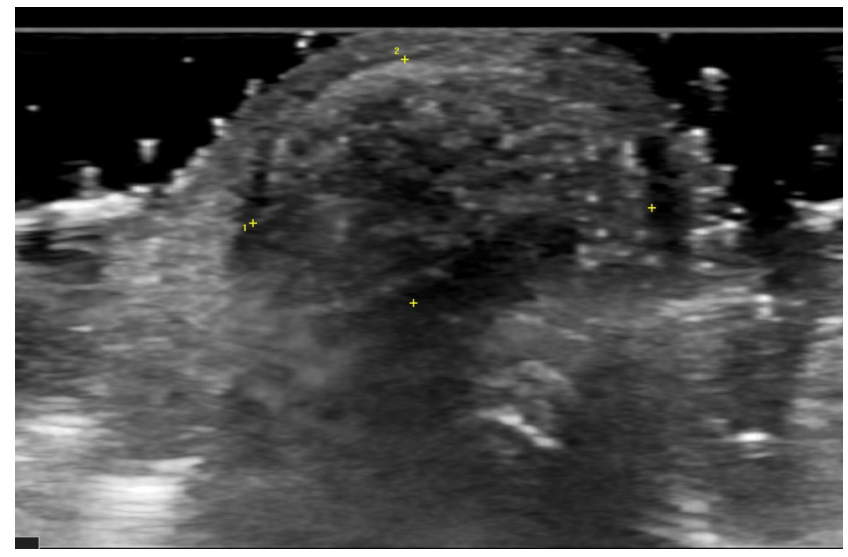

Fig. 2. Preoperative ultrasonography image showing a $1.15 \times 0.7 \times$ $1.45-\mathrm{cm}$ heterogenic hyperechoic mass on the auricle.

A diagnosis of tumoral calcinosis was confirmed. The patient was discharged following surgery, with no complaint of discomfort. The patient's lab values were serum phosphorus 4.7 $\mathrm{mg} / \mathrm{dL}$ and serum calcium $9.4 \mathrm{mg} / \mathrm{dL}$, all within normal limits. After 9 months of follow-up, there were no signs of recurrence of the tumor (Fig. 5) and the patient was highly satisfied with the surgical results. We obtained the patient's medical records and reviewed the related literature. This study was approved by the Chonnam National University Hospital Institutional Review Board (IRB No. CNUH-2018-209).

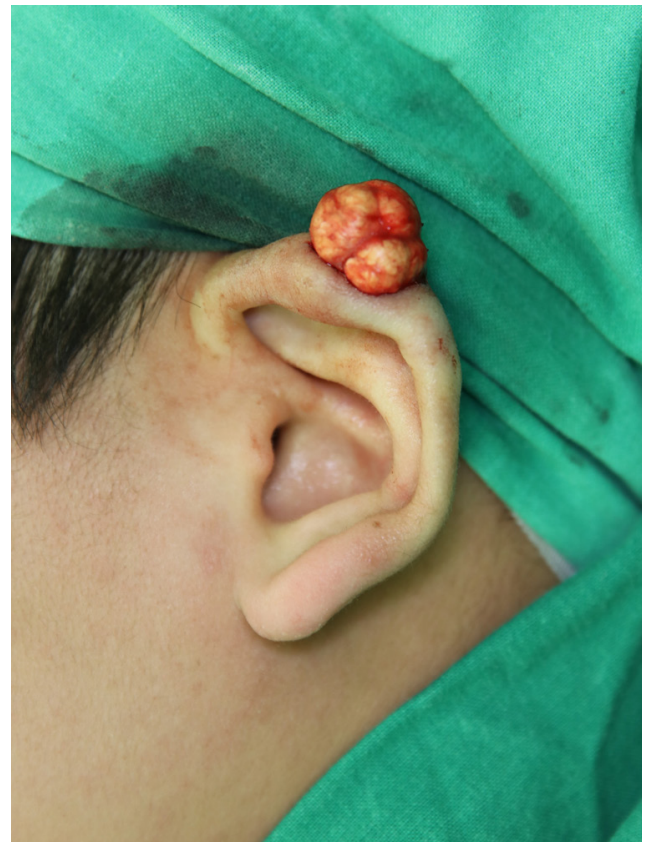

Fig. 3. Intraoperative photograph of the auricular mass.
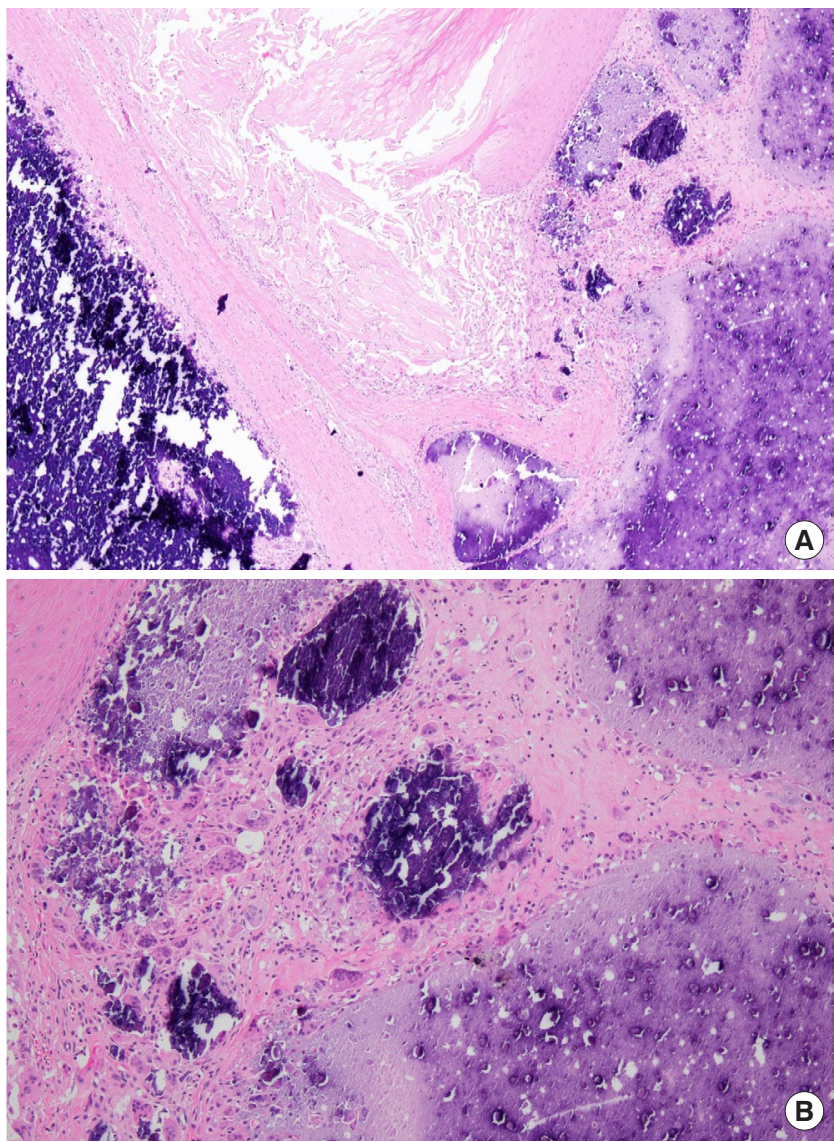

Fig. 4. Pathological examination of the specimen. (A) Multiple cystic spaces are formed and calcification is in progress with eosinophilic debris $(\mathrm{H} \& \mathrm{E}, \times 40)$. (B) Foreign body giant cell reaction with calcification and epithelioid cell $(\mathrm{H} \& \mathrm{E}, \times 100)$ can be observed. 


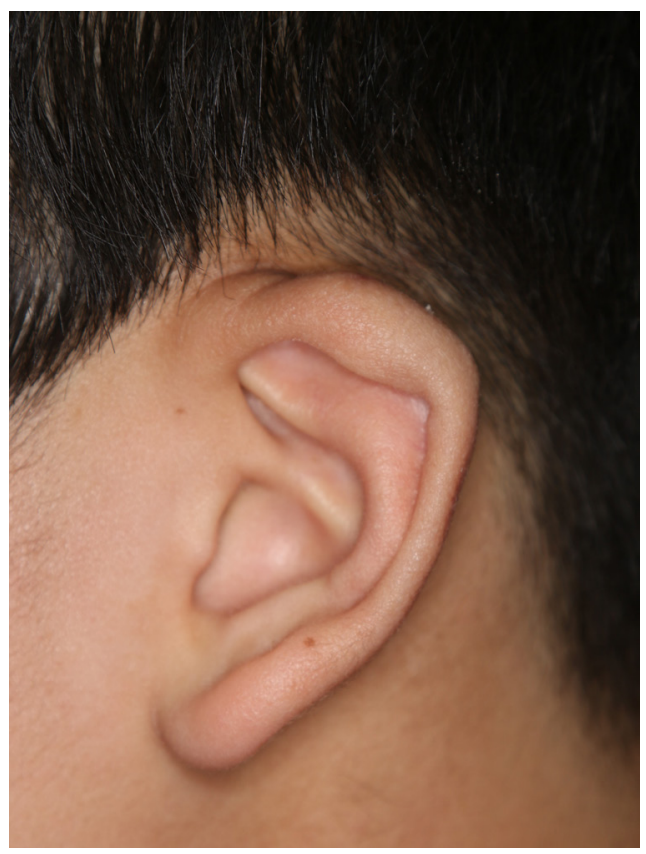

Fig. 5. Postoperative photograph at 9 months.

\section{DISCUSSION}

Tumoral calcinosis is classified into three subtypes: primary normophosphatemic tumoral calcinosis, primary hyperphosphatemic tumoral calcinosis, and secondary tumoral calcinois. The first subtype often shows familial tendency and is most commonly seen in African patients. It also occurs sporadically and is associated with past trauma. The second subtype is a rare autosomal recessive disorder caused by mutation of the GALTN3 gene. The third subtype presents calcium deposits at multiple sites due to renal failure or renal dialysis. Normophosphatemic tumoral calcinosis has a better prognosis than other subtypes. Additionally, as each subtype has a different response to treatment, it is advisable to identify the subtype correctly [3,5-7].

In case of suspected tumoral calcinosis, plain radiographs can be taken to confirm amorphous calcification. Investigations like ultrasonography, computed tomography, etc., can aid in identifying the correct size and surgical planning. Magnetic resonance imaging scans show inhomogeneous, high-signal intensity on T2-weighted images and homogeneous, low-signal intensity on T1-weighted images [3,5,8]. Furthermore, serum calcium levels, serum phosphorus levels, serum parathormone levels, 1,25-dihydroxy-vitamin D levels, and renal function tests should be checked to classify the disease.

Other benign non-epithelial tumors of the ear auricle include cyst, keloid, hemangioma, hematoma, osteoma, and soft tissue chondroma, while malignant nonepithelial tumors include chondrosarcoma [9-11]. Therefore, a definitive diagnosis is needed and histological examination becomes essential. Histopathological examination of this case shows extensive calcification with surrounding dense hyalinized stroma. Foreign body giant cell reaction is also observed and epithelioid cells are seen in many cases [3]. Additionally, there was no history of trauma, specific reaction to foreign body, metabolic disorder, nor any significant family history, and the serum biochemical level was normal. Therefore, it was diagnosed as tumoral calcinosis, a subtype of idiopathic calcinosis.

The standard treatment modality for tumoral calcinosis is early surgical excision $[3,5]$. However, when there is a mass on the joint, recurrence rates tend to be higher in adults. Hence, in normo- or hyperphosphatemic cases, treatment with phosphate-binding chelating agents or acetazolamide may help to reduce recurrence rate of tumoral calcinosis. With accompanying renal failure, phosphorus restricted diet, dialysis, and phosphate-binding agents are the preferred treatment modalities [3]. On the other hand, surgical treatments such as parathyroidectomy or renal transplantation may be considered if the serum biochemical level is not under control.

Our study had the limitation that tumoral calcinosis of the auricle has not yet been established with respect to its nature, recurrence, and prognosis. Therefore, further studies and longterm data are required to establish biological understanding and treatment for patients. There are several possible tumors of the auricle, among which tumoral calcinosis is extremely rare and can be misdiagnosed as other tumors. Hence, accurate diagnosis is essential for therapy. Serum calcium, serum phosphorus, and gene analysis tests may also be performed when necessary. Surgical resection should be considered as the treatment of choice.

\section{CONFLICT OF INTEREST}

KSK, a member of the Archives of Craniofacial Surgery, is the author of this article. However, he played no role whatsoever in the editorial evaluation of this article or the decision to publish it. Except for that, no potential conflict of interest relevant to this article was reported.

\section{PATIENT CONSENT}

The parents of the patient provided written informed consent for the publication and the use of his images.

\section{ORCID}

Sam Yong Lee https://orcid.org/0000-0002-3185-2519 
Han Gyeol Lee https://orcid.org/0000-0001-9886-1738

Kwang Seog Kim https://orcid.org/0000-0002-6766-4640

Jae Ha Hwang https://orcid.org/0000-0001-6992-8067

\section{REFERENCES}

1. Muddegowda PH, Lingegowda JB, Ramachandrarao RK, Konapur PG. Calcinosis cutis: report of 4 cases. J Lab Physicians 2011;3:125-6.

2. Laguna EV, Martinez AA, Burgos F. Petrified ear: a case of calcinosis cutis. Acta Derm Venereol 2009;89:527-8.

3. Fathi I, Sakr M. Review of tumoral calcinosis: a rare clinicopathological entity. World J Clin Cases 2014;2:409-14.

4. Inclan A, Leon P, Camejo MG. Tumoral calcinosis. JAMA 1943;121:490-5.

5. Kim YD, Woo YK, Song SY, Bae CH. A case of tumoral calcinosis at the auricle. Korean J Otorhinolaryngol Head Neck
Surg 2009;52:985-7.

6. Booth GS, Vithana RE, DeMello DE, Mandell GA. Recurrent idiopathic tumoral calcinosis in a child postoperatively. Radiol Case Rep 2015;7:735.

7. Zvaigzne CG, Patton DJ, Kaur H, Trevenen CL, Kaura D. Subscapular tumoral calcinosis in a toddler: case report. J Radiol Case Rep 2012;6:10-7.

8. del Puerto Troncoso C, Tuma MC, Bombardiere SG, Silva-Valenzuela S. Bilateral ossification of the auricular cartilage. Actas Dermosifiliogr 2015;106:433-5.

9. Kang YJ, Park JM, Han JJ, Park SN. A case of idiopathic calcinosis cutis on auricle. J Korean Skull Base Soc 2017;12:42-6.

10. Sand M, Sand D, Brors D, Altmeyer P, Mann B, Bechara FG. Cutaneous lesions of the external ear. Head Face Med 2008;4:2.

11. Csssisi NJ. Neoplasms of the auricle. In: Cummings CW, Schuller DE, editors. Otolaryngology-head and neck surgery. 2nd ed. St. Louis: Mosby-Year Book; 1993. p. 2965. 